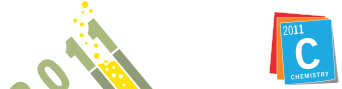

International Year of

\title{
VALIDAÇÃO DE MÉTODO ANALÍTICO POR CROMATOGRAFIA LÍQUIDA DE ALTA EFICIÊNCIA PARA DOSEAMENTO DO ADAPALENO EM SUSPENSÕES DE NANOCÁPSULAS
}

\author{
Jerusa Goi Barrios, Gabriela D’Avila Farias*, Isabel Roggia, Sandra Cadore Peixoto, Flábio R. Pons, Márcia Bruschi, \\ Renata Platcheck Raffin e Marta Palma Alves \\ Departamento de Nanociências, Centro Universitário Franciscano, Rua dos Andradas, 1614, 97010-032 Santa Maria - RS, Brasil
}

Recebido em 27/10/10; aceito em 22/2/11; publicado na web em 12/5/11

\begin{abstract}
VALIDATION OF ANALYTICAL METHOD BY HPLC FOR DETERMINATION OF ADAPALENE IN SUSPENSIONS OF NANOCAPSULES. Adapalene is a retinoid drug often used for disorders of the skin, mainly acne. In this work, an analytical method for the quantification of adapaleno in suspensions of nanocapsules by HPLC was developed and validated. The method was linear in the range of $10-30 \mu \mathrm{g} \mathrm{mL}^{-1}$, with a good correlation coefficient $(\mathrm{r}=0.994)$. Precision and accuracy analysis showed low relative standard deviation $(<4.6 \%)$ and a good recovery percentual $(98.2-106.9 \%)$. The procedure was specific, linear, precise, exact and robust so that the method can be applied in quantification of adapalene in suspensions of nanocapsules.
\end{abstract}

Keywords: adapalene; validation; HPLC.

\section{INTRODUÇÃO}

Com o nome químico ácido 6-[3-(1-adamantil)-4-metoxifenil]2-naftoico, fórmula molecular $\mathrm{C}_{28} \mathrm{H}_{28} \mathrm{O}_{3}$ e massa molar de $412,52 \mathrm{~g}$ $\mathrm{mol}^{-1},{ }^{1} \mathrm{o}$ adapaleno (Figura 1) é um pó branco solúvel em tetraidrofurano, ligeiramente solúvel em etanol e praticamente insolúvel em água. ${ }^{2}$ Este fármaco é um retinoide de terceira geração derivado do ácido naftoico, ${ }^{3}$ que possui atividade comedolítica e anti-inflamatória apropriada, sendo uma alternativa tópica para o tratamento da acne. ${ }^{4}$ A acne é uma doença genético-hormonal, ${ }^{5}$ que envolve a unidade pilossebácea, sendo mais frequente e intensa em áreas onde as glândulas sebáceas são maiores e mais numerosas, como na face, no tórax e dorso. ${ }^{6}$<smiles>COc1ccc(-c2ccc3cc(C(=O)O)ccc3c2)cc1C12CC3CC(CC(C3)C1)C2</smiles>

Figura 1. Estrutura molecular do adapaleno

O adapaleno apresenta alta estabilidade frente à luz e ao oxigênio, não demonstrando reatividade química. ${ }^{1,7}$ Esse fármaco penetra na unidade pilossebácea podendo apresentar alguns efeitos indesejáveis, sendo a irritação e o ressecamento da pele os mais comuns. ${ }^{4,8,9} \mathrm{Em}$ vista disso, busca-se com o desenvolvimento de sistemas nanoestruturados minimizar esses efeitos adversos e aumentar a eficácia desse ativo direcionando-o ao local de ação.

Através desta abordagem nanotecnológica, foram desenvolvidas suspensões contendo nanocápsulas poliméricas de adapaleno utilizando óleo de melaleuca como núcleo oleoso e poli-( -caprolactona) como unidade polimérica. No entanto, a extração do fármaco a partir deste sistema nanoestruturado para fins de quantificação é extremamente difícil, sendo necessária a utilização de uma mistura de solven-

\footnotetext{
*e-mail: gabiifarias@ hotmail.com
}

tes que possibilite a solubilização da parede polimérica, bem como do ativo. ${ }^{10}$ Assim sendo, o desenvolvimento de um método simples, rápido e preciso para a extração e quantificação do adapaleno a partir das nanocápsulas é altamente desejável. Em vista disso, o presente trabalho teve como objetivo desenvolver e validar um método analítico para quantificação do adapaleno através de cromatografia líquida de alta eficiência (CLAE).

Para garantir que um novo método analítico gere informações confiáveis e interpretáveis sobre a amostra, ele deve passar por um processo de avaliação denominado validação, que garante a confiabilidade e rastreabilidade dos resultados. ${ }^{11} \mathrm{~A}$ validação deve garantir, através de estudos experimentais, que o método atenda às exigências das aplicações analíticas, assegurando a confiabilidade dos resultados. ${ }^{12}$

Vale ressaltar que a literatura consultada não apresenta método analítico validado para a determinação do adapaleno.

\section{PARTE EXPERIMENTAL}

\section{Materiais}

Para a formação das nanocápsulas foi utilizado adapaleno (teor $=$ 100,3\%) (Pharma Nostra ${ }^{\circledR}$ ), monoestearato de sorbitano (Sigma Aldrich $^{\circledR}$ ), óleo de melaleuca (Via Farma ${ }^{\circledR}$ ), poli-( -caprolactona) massa molecular $=65000\left(\right.$ Aldrich $\left.^{\circledR}\right)$, polissorbato $80\left(\right.$ Via Farma $\left.^{\circledR}\right)$ e acetona P.A $\left(\right.$ Nuclear $\left.^{\circledR}\right)$. Os solventes empregados foram acetonitrila grau CLAE (J.T. Baker ${ }^{\circledR}$ ), ácido fosfórico P.A (Nuclear $\left.{ }^{\circledR}\right)$, metanol grau CLAE (J.T. Baker ${ }^{\circledR}$ ) e tetra-hidrofurano grau CLAE (J.T. Baker ${ }^{\circledR}$ ).

\section{Equipamentos}

Utilizou-se cromatógrafo líquido de alta eficiência Shimadzu YL9100 (CLAE) equipado com bomba modelo LC-10AD, detector com comprimento de onda variável UV/Vis modelo SPD-10Avp, controlador SLC-10Avp, integrador automático computadorizado com software Class VP ${ }^{\circledR}$ e injetor automático SIL-10-Avp, forno para coluna CTO-10Asvp Shimadzu e evaporador rotatório 801 (Fisatom $^{\circledR}$ ). 


\section{Preparo das suspensões de nanocápsulas contendo adapaleno}

As nanocápsulas contendo adapaleno foram preparadas através do método de deposição interfacial do polímero pré-formado. ${ }^{13} \mathrm{~A}$ fase orgânica, composta de acetona, adapaleno, poli-(ع-caprolactona), monoestearato de sorbitano e óleo de melaleuca, foi vertida na fase aquosa, contendo polissorbato 80 , sob agitação magnética moderada. $\mathrm{O}$ volume da suspensão obtida foi reduzido utilizando-se evaporador rotatório, de maneira que a concentração final de adapaleno foi ajustada para $0,3 \mathrm{mg} \mathrm{mL}^{-1}(\mathrm{~m} / \mathrm{v})$.

\section{Extração do fármaco das nanocápsulas}

Após o preparo das suspensões, as amostras foram tratadas com acetonitrila (ACN), tetra-hidrofurano (THF) e metanol (2,5:5,0:2,5 $\mathrm{v} / \mathrm{v} / \mathrm{v}$ ) com o objetivo de dissolver o polímero e o fármaco. Posteriormente, as mesmas foram filtradas em membrana de poliacetilamida com porosidade de $0,45 \mu \mathrm{m}$.

\section{Parâmetros cromatográficos}

As condições cromatográficas foram otimizadas para determinação do adapaleno nas suspensões de nanocápsulas e estão descritas na Tabela 1.

Tabela 1. Condições cromatográficas usadas na quantificação do adapaleno em suspensões contendo $\mathrm{NC}^{*}$

\begin{tabular}{ll}
\hline Característica & Descrição \\
\hline Coluna & Lichropher $^{\circledR} 100 \mathrm{RP}-18,250 \mathrm{~mm}, 4 \mathrm{~mm}, 5 \mu \mathrm{m}-$ Merck $^{\circledR}$ \\
Pré-coluna & Mesmo material da coluna Lichropher ${ }^{\circledR}$ Merck $^{\circledR}$ \\
Fluxo & $1,0 \mathrm{~mL} \mathrm{~min}{ }^{-1}$ \\
Volume de injeção & $20,0 \mu \mathrm{L}$ \\
Detecção & $254 \mathrm{~nm}$ \\
Fase móvel & Metanol:água $(95: 5, \mathrm{v} / \mathrm{v})$, acidificada a pH 3,0 com $^{\mathrm{H}_{3} \mathrm{PO}_{4}(1: 1, \mathrm{v} / \mathrm{v})}$ \\
\hline
\end{tabular}

*NC: nanocápsulas

\section{Validação do método analítico}

A validação do método analítico foi realizada segundo os critérios propostos pela resolução da ANVISA RE $n^{\circ} 899$, de 29/5/2003 ${ }^{12}$ e pela ICH (International Conference on Harmonization) $)^{14}$ de 2005 . Os parâmetros avaliados no desenvolvimento deste trabalho foram: especificidade, linearidade, precisão intermediária, repetibilidade, robustez, exatidão, limite de detecção e limite de quantificação.

A especificidade do método foi avaliada através de análises comparativas entre as suspensões de nanocápsulas sem adapaleno (branco) e as suspensões contendo o fármaco na concentração de $20,0 \mu \mathrm{g} \mathrm{mL} \mathrm{m}^{-1}$.

Para o estudo da linearidade foram preparadas soluções para a construção das curvas de calibração, analisadas em 3 dias diferentes e com leituras em triplicata, em 5 concentrações de adapaleno $(10,0$; 15,$\left.0 ; 20,0 ; 25,0 ; 30,0 \mu \mathrm{g} \mathrm{mL}^{-1}\right)$.

A precisão foi avaliada através da análise de 6 soluções amostra (suspensão de nanocápsulas contendo adapaleno) na concentração de 20,0 $\mu \mathrm{g} \mathrm{mL} \mathrm{m}^{-1}$ em um único dia (repetibilidade) e através da precisão intermediária com injeções das soluções $\left(10,0 ; 20,0\right.$ e 30,0 $\left.\mu \mathrm{g} \mathrm{mL}^{-1}\right)$, em dias diferentes. A robustez do método foi verificada variando-se o analista durante o ensaio de precisão.

A exatidão do método foi determinada adicionando-se uma quantidade conhecida de fármaco $\left(10,0 \mu \mathrm{g} \mathrm{mL}^{-1}\right)$ à solução amostra, resultando nas concentrações 10,$0 ; 20,0$ e $30,0 \mu \mathrm{g} \mathrm{mL}^{-1}$.
Os limites de detecção (LOD) e de quantificação (LOQ) foram calculados através da divisão do desvio padrão dos coeficientes lineares das curvas de calibração do ensaio de linearidade pela média dos coeficientes angulares destas respectivas curvas, multiplicados por 3 e 10 , respectivamente, conforme sugerido pela $\mathrm{ICH} .{ }^{14,15}$

\section{RESULTADOS E DISCUSSÃO}

\section{Parâmetros cromatográficos}

A técnica de extração do adapaleno a partir das suspensões, bem como as condições cromatográficas desenvolvidas foram consideradas adequadas para o doseamento do adapaleno em suspensões das nanocápsulas (NC).

\section{Validação do método analítico}

A validação de métodos analíticos já foi desenvolvida para formas farmacêuticas de liberação modificada. ${ }^{16-19}$

O método analítico validado foi específico para este tipo de análise e encontra-se em concordância com as especificações oficiais da área.

A Figura 2a ilustra um cromatograma de uma amostra branco e a Figura 2 b destaca um cromatograma do adapaleno extraído da suspensão de NC na concentração $20,0 \mu \mathrm{g} \mathrm{mL} \mathrm{m}^{-1}$.
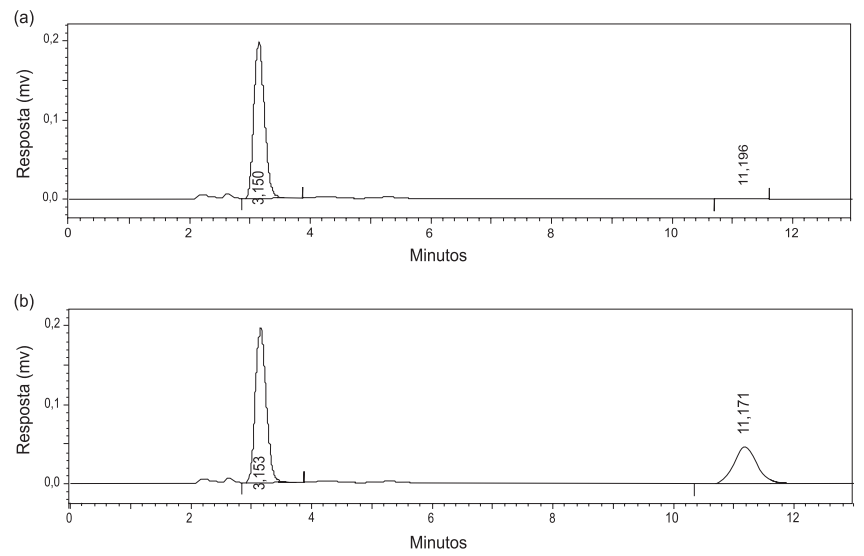

Figura 2. Cromatogramas da suspensão de nanocápsulas vazias (a) e contendo adapaleno (b) nas condições cromatográficas desenvolvidas, conforme descrito na Tabela 1

Vale ressaltar que a amostra branco foi preparada da mesma forma que as suspensões de nanocápsulas contendo adapaleno, porém sem a adição do fármaco.

Conforme cromatograma demonstrado na Figura 2a, pode-se observar que no tempo de retenção do adapaleno (11 min) não há sinal cromatográfico, constatando-se então que esta pode ser utilizada como controle para detecção do ativo.

De acordo com a Figura 2b, tem-se o sinal do adapaleno na concentração de 20,0 $\mu \mathrm{g} \mathrm{mL}^{-1}$ bem definido (em tempo de retenção de $11 \mathrm{~min}$ ), o que resulta em boa sensibilidade do método de extração e de análise desenvolvido.

Considerando que o tempo de retenção do adapaleno, nas condições cromatográficas otimizadas neste estudo, é de 11 min e que em 3,1 min tem-se a eluição do THF, vale ressaltar que este intervalo de separação entre os dois compostos é necessário, visto que a aproximação da eluição, conforme testes preliminares realizados, diminui a especificidade do método.

Para o ensaio de linearidade, a solução mãe foi preparada na concentração de $2,5 \mathrm{mg} \mathrm{mL}^{-1}$, adicionando-se os solventes THF, metanol e acetonitrila. Posteriormente, as respectivas diluições des- 
critas na Tabela 2 foram preparadas em metanol, para a construção da curva analítica.

Tabela 2. Valores de DPR para as diferentes concentrações da curva analítica

\begin{tabular}{cc}
\hline Concentração $\left(\mu \mathrm{g} \mathrm{mL}^{-1}\right)$ & DPR $(\%)$ \\
\hline 10,0 & 1,07 \\
15,0 & 3,07 \\
20,0 & 3,97 \\
25,0 & 2,39 \\
30,0 & 2,73 \\
\hline
\end{tabular}

Com isso, a linearidade do método foi avaliada a partir da injeção das curvas analíticas em triplicata, em 3 dias diferentes e em 5 concentrações de adapaleno. Para cada concentração foi calculado o desvio padrão relativo (DPR) em relação às 9 curvas analisadas (Tabela 2).

Pode-se observar que os valores de DPR para as diferentes concentrações foram inferiores a 4,0\%.

Para a avaliação da linearidade, a equação da reta $(\mathrm{y}=71875 \mathrm{x}$ - 90836) foi obtida através de estudos de regressão linear, entre a concentração de adapaleno e suas respectivas respostas, obtendo-se um coeficiente de determinação de 0,994 , o que se caracteriza linear e adequado para tal uso.

O estudo da linearidade do método analítico foi realizado através de análise de variância, verificando-se regressão linear significativa e desvio da linearidade não significativo $(\mathrm{p}<0,01)$ com injeção de concentrações compreendidas entre 10,0 e $30,0 \mu \mathrm{g} \mathrm{mL} \mathrm{mL}^{-1}$, em triplicata.

A partir dos resultados obtidos no desenvolvimento do método, conclui-se que a curva analítica pode ser utilizada para a quantificação dos valores experimentais do adapaleno, visto que o coeficiente de determinação foi maior que 0,99 , atestando a qualidade da curva analítica obtida, pela baixa dispersão do conjunto de pontos experimentais. ${ }^{11,12,14,15}$

A repetibilidade ou precisão intradia do método foi avaliada a partir da determinação do desvio padrão relativo (DPR) intradia, a precisão intermediária ou precisão interdias foi determinada a partir dos valores de DPR interdias e a robustez do método foi avaliada através da análise dos resultados obtidos por analistas diferentes. Estes valores de DPR estão descritos nas Tabelas 3 e 4, que definem os valores de recuperações obtidos para os ensaios de repetibilidade, precisão intermediária e robustez para a quantificação do adapaleno por CLAE.

Na Tabela 3 pode-se observar que nas 6 medidas para o ensaio de repetibilidade os valores de recuperação ficaram entre 98,2 e 106,9\% e os valores de DPR entre 0,2 e 4,9, o que demonstra conformidade com os parâmetros estabelecidos para a validação de métodos cromatográficos em fármacos. ${ }^{12}$

Nos ensaios intradia ( $\left.1^{\circ} \mathrm{dia}\right)$, para o analista 1 os valores de recuperação ficaram entre 98,4 e 99,4\%, e para o analista 2 entre 92,4

Tabela 3. Valores das recuperações obtidas no ensaio de repetibilidade para a quantificação do adapaleno por CLAE

\begin{tabular}{ccc}
\hline Repetições & $\mathrm{R}(\%)^{*}$ & $\mathrm{DPR}(\%)^{* *}$ \\
\hline N1 & 98,4 & 0,6 \\
N2 & 106,9 & 0,3 \\
N3 & 103,8 & 0,2 \\
N4 & 98,3 & 4,9 \\
N5 & 103,8 & 0,5 \\
N6 & 98,2 & 3,0 \\
\hline
\end{tabular}

*Valores de recuperação do adapaleno; **valores de desvio padrão relativo das medidas; $\mathrm{N}=$ número de réplicas
Tabela 4. Valores de recuperação para os ensaios de precisão intermediária e robustez obtidos por analistas diferentes para a quantificação do adapaleno em CLAE

\begin{tabular}{|c|c|c|c|c|c|c|c|}
\hline \multirow[t]{2}{*}{ Dia } & \multirow{2}{*}{$\begin{array}{l}\text { Concentração } \\
\left(\mu \mathrm{g} \mathrm{mL} L^{-1}\right)\end{array}$} & \multicolumn{2}{|c|}{$\begin{array}{c}\text { Analista } 1 \\
\text { Teor da amostra }\end{array}$} & \multicolumn{2}{|c|}{$\begin{array}{c}\text { Analista } 2 \\
\text { Teor da amostra }\end{array}$} & \multirow{2}{*}{$\begin{array}{c}\text { Média do } \\
\text { dia } \pm \text { DP* } \\
(\%)\end{array}$} & \multirow{2}{*}{$\begin{array}{c}\text { DPR do } \\
\text { dia** } \\
(\%)\end{array}$} \\
\hline & & $\left(\mu \mathrm{g} \mathrm{mL} L^{-1}\right)$ & $(\%)$ & $\left(\mu \mathrm{g} \mathrm{mL} L^{-1}\right)$ & $(\%)$ & & \\
\hline \multirow{3}{*}{$1^{\circ}$ dia } & 10,0 & 9,8 & 98,4 & 9,5 & 95,5 & \multirow{3}{*}{$98,4 \pm 4,4$} & \multirow{3}{*}{4,5} \\
\hline & 20,0 & 19,8 & 99,4 & 21,1 & 105,8 & & \\
\hline & 30,0 & 29,6 & 98,8 & 27,7 & 92,4 & & \\
\hline \multirow{3}{*}{$2^{\circ}$ dia } & 10,0 & 10,6 & 106,4 & 1,0 & 100,3 & \multirow{3}{*}{$\begin{array}{c}101,2 \pm \\
3,3\end{array}$} & \multirow{3}{*}{3,2} \\
\hline & 20,0 & 20,1 & 100,6 & 19,8 & 99,1 & & \\
\hline & 30,0 & 31,1 & 103,8 & 29,1 & 97,1 & & \\
\hline
\end{tabular}

*DP: Desvio padrão; **DPR: desvio padrão relativo

e 105,8\%, com valores de DPR de 4,5\%. No $2^{\circ}$ dia de realização do ensaio, para o analista 1 os valores de recuperação ficaram entre 100,6 e $106,4 \%$ e para o analista 2 entre 97,1 e $100,3 \%$ com DPR de $3,2 \%$.

Com isso, a precisão foi avaliada pela aproximação dos resultados obtidos em uma mesma amostra, sendo que os valores de DPR determinados intradia e interdias foram todos menores que o valor máximo aceitável preconizado pela ANVISA ${ }^{12}$ como $5 \%$, demonstrando que o método possui precisão e repetibilidade adequadas.

Além disso, pode-se observar que o método manteve-se robusto, indicando confiabilidade dos resultados, visto que a mudança de analista não influenciou de forma significativa os resultados obtidos.

Os resultados percentuais de exatidão do método obtidos estão dispostos na Tabela 5.

Tabela 5. Valores experimentais da exatidão do método

\begin{tabular}{ccccccc}
\hline \multirow{2}{*}{$\begin{array}{c}\text { Concentração } \\
\left.(\mu \mathrm{g} \mathrm{mL})^{-1}\right)\end{array}$} & \multicolumn{2}{c}{$1^{\circ}$ dia } & \multicolumn{2}{c}{$2^{\circ}$ dia } & \multicolumn{2}{c}{$3^{\circ}$ dia } \\
\cline { 2 - 7 }$(\%)$ & DPR $(\%)$ & $\mathrm{R}(\%)$ & DPR $(\%)$ & $\mathrm{R}(\%)$ & $\mathrm{DPR}(\%)$ \\
\hline 10,0 & 99,5 & 0,4 & 99,1 & 2,0 & 99,7 & 2,7 \\
20,0 & 100,9 & 1,4 & 100,7 & 1,7 & 101,1 & 1,4 \\
30,0 & 101,2 & 2,7 & 101,4 & 2,3 & 100,4 & 2,9 \\
\hline
\end{tabular}

Os valores descritos na Tabela 5 foram satisfatórios, pois todas as percentagens de recuperação ficaram entre 99,1 e 101,4\%, demonstrando conformidade com os limites estabelecidos pela $\mathrm{ICH},{ }^{14}$ que são de 98 a 102\%. Em vista disso, o método pode ser caracterizado como tendo uma boa exatidão.

Os valores obtidos para os limites de detecção e de quantificação foram 0,11 e $0,37 \mu \mathrm{g} \mathrm{mL} \mathrm{m}^{-1}$, respectivamente, indicando uma boa sensibilidade do método para a determinação do adapaleno. Visto que a concentração do fármaco nas suspensões de nanocápsulas é $0,03 \%$ e nos geis comerciais é $0,1 \%,{ }^{20,21}$ é então possível determinar a concentração do adapaleno nos dois casos.

\section{CONCLUSÃO}

O método analítico de detecção e quantificação do adapaleno, validado segundo ANVISA (2003) e ICH (2005), mostrou-se linear, exato, preciso, específico e robusto na faixa de concentração de 10,0 a $30,0 \mu \mathrm{g} \mathrm{mL} \mathrm{mL}^{-1}$. Foram determinados também os limites de quantificação e de detecção, indicando que o método é eficiente para quantificar o adapaleno em concentração mínima de $0,37 \mu \mathrm{g} \mathrm{mL}^{-1}$.

Desta forma, pode-se concluir que o método validado se mostrou adequado para a quantificação do adapaleno em suspensões de nanocápsulas, apresentando também sensibilidade apropriada para quantificação em outras formas farmacêuticas. 


\section{REFERÊNCIAS}

1. Irby, C. E.; Yentzer, B. A.; Felsman S. R.; J. Adol. Health. 2008, 43, 421.

2. Piskin, S.; Uzunali, E.; Ther. Clin. Risk Manag. 2007, 3, 621.

3. Trichard, L.; Delgado-Charro, M. B.; Guy, R. H.; Fattal, E.; Bochot A.; Pharm. Res. 2008, 25, 435.

4. Chivot, M.; Am. J. Clin. Dermatol. 2005, 6, 13.

5. Olutunmbi, Y.; Paley, K.; English, J. C.; J. Pediatr. Adolesc. Gynecol. 2008, 21, 171 .

6. Silva, P.; Farmacologia, 6 ${ }^{\mathrm{a}}$ ed., Ed. Guanabara: Rio de Janeiro, 2002.

7. Shroot, B.; Michel, S.; J. Am. Acad. Dermatol. 1997, 36, 96.

8. Korolkovas, A.; Dicionário Terapêutico Guanabara, $15^{\mathrm{a}}$ ed., Ed. Guanabara: Rio de Janeiro, 2009.

9. Lima, D. R.; Manual de Farmacologia Clínica Terapêtica e Toxicologia, 1ª ed., Ed. Guanabara: Rio de Janeiro, 2004.

10. Paese, K.; Jaeger, A.; Poletto, F. S.; Pinto, E. F.; Rossi-Bergmann, B.; Pohlmann, A. R.; Guterres, S. S.; J. Biomed. Nanotechnol. 2009, 5, 240.

11. Ribani, M.; Bottoli, C. B.; Collins, C.; Jardim, I. C.; Melo, L.; Quim. Nova 2004, 27, 771 .
12. Agência Nacional de Vigilância Sanitária - ANVISA; RE no 899 de 29/05/2003: Guia para validação de métodos analíticos e bioanalíticos, Ministério da Saúde: Brasil 2003.

13. Fessi, H.; Puiseiux, F.; Devissaguet, J-P.; Eur. Pat., 0274961 A1 1988.

14. ICH - International Conference on Harmonization; Q2R1-validation of Analytical procedure: Text and Methodology, 2005.

15. ICH - International Conference on Harmonization of Technical Requeriments for registration of Pharmaceutical for Human use; $Q 2 B$-validation of Analytical procedure: methodology, 1996.

16. Barboza, F. M.; Dalla Vecchia, D.; Pereira, A. V.; Stulzer, H. K.; Silva, M. A. S.; Quim. Nova 2010, 33, 747.

17. Raffin, R. P.; Colomé, L. M.; Guterres, S. S.; Pohlmann, A. R.; Quim. Nova 2007, 30, 1001.

18. Moraes, C. M.; De Paula, E.; Rosa, A. H.; Fraceto, L. F.; Quim. Nova 2008, 31, 2152.

19. Grillo, R.; De Melo, N. F. S.; De Araújo, D. R.; De Paula, E.; Dias Filho, N. L.; Rosa, A. H.; Fraceto, L. F.; Lat. Am. J. Pharm. 2009, 28, 393.

20. Galvin, S. A.; Gilbert, R.; Baker, M.; Guibal, F.; Tuley, M. R.; Br. J. Dermatol. 1998, 139, 34.

21. Waugh, J.; Noble, S.; Scott, L. J.; Am. J. Clin. Dermatol. 2004, 5, 369. 